\section{Project VISUAL: Facilitating the Connection Between Art and Science}

\author{
L.M. Strzegowski* and T.P. Russell \\ University of Massachusetts Amherst \\ mrsec@polysci.umass.edu
}

Nearly five years ago, Representative Vernon Ehlers, in his report to Congress on a House of Representatives study entitled "Unlocking Our Future: Toward a New National Science Policy, noted that the American public does not understand science and its practice. A major recommendation that emerged from this study was the need to "make scientists socially responsible." This sentiment was echoed in a National Research Council's report, "Materials in a New Era," where Neal Lane, former Director of the National Science Foundation, was quoted as saying, "It is necessary to involve material scientists in a new role, undoubtedly an awkward one for many, that might be called the civic scientist'." Why the concern? The answer is clear. "Our prosperity, security, and health depend directly on the educational achievement of all students, not only those who will become scientists and engineers, but all workers, voters, parents, and consumers., ${ }^{\text {"3 }}$ Yet, if we add to this the continual reduction in the level of students engaging in scientific careers ${ }^{4}$, it is apparent that the excitement and significance of scientific research is not reaching the general public or aspiring students. What can be done?

VISUAL (Ventures in Science Using Art Laboratory), a recently launched educational outreach program of the Materials Research Science and Engineering Center on Polymers (MRSEC) at the University of Massachusetts Amherst, is a different approach to effect a change. The program is based on the premise that the visual arts can serve as an effective means to stimulate, educate and promote materials science research to the general public and to students of all ages. All of us engaged in scientific research are frequently, perhaps even

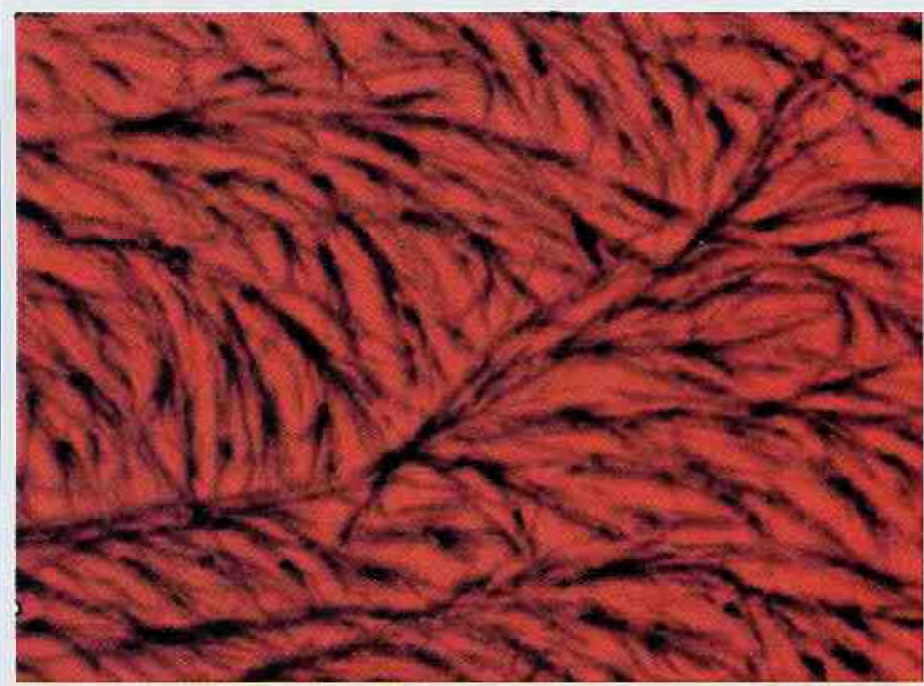

Dmytro's Quantum Forest The patterns that can be produced by a fluid, like oil or water, on a non-stick surface will depend upon the amount of liquid and how much the liquid is repelled from the surface. If you could adjust the level of stickiness or, in other words, control the surface tension, you can control the type of pattern that is formed. Dmytro Nykypanchuk, a graduate student, using fuorescent particles, was able to arrange the particles in tree-like formations where each limb contains thousands of the particles arranged in rows. While the arrangement of the particles can be seen, each of the individual particles is too small to be observed with your eye. However, the type of patterne that is formed is related to the strength of the interactions of the liquid in which the particles are dispersed and the surface of the tunderlying support. OUniversity of Massachusetts Amherst, 2003.

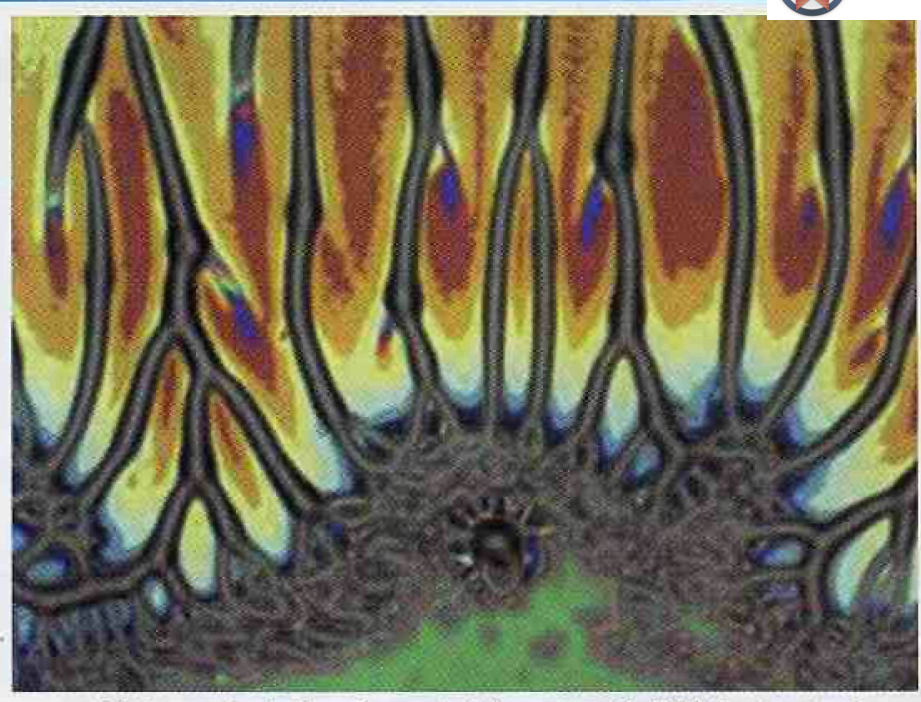

Metamorphosis By using an electric or magnetic field, the structure in a polymer film can be forced into an unstable condition. If, in addition, the polymer does not like the surface of the underlying support, the polymer will try to withdraw from the surface, like water in a Teflon pan. The combination of these two conditions causes an instability, which was captured by $T$ ing $X u, a$ graduate student, with an optical microscope. By using the interference colors, the changes in the film thickness can be determined. The instability, in this case, has caused the formation of an unusual structure that changes size as it goes across the fim. The film is unstable at one point and gradually changes to a stable film. In essence, the film has undergone a metamorphosis from being stable to unstable, OUniversity of Massachusetts Anherst, 2003.

daily, exposed to imagery that is not only breathtaking to behold, but contains fundamental information that is key to understanding specific phenomena or underlying scientific principles. VISUAL uses this imagery to provide a unique avenue by which an interest and appreciation of scientific research can be nurtured.

Consider the images shown in this article, which span topics from nanoparticles to thin films of block copolymers. The sheer beauty of the optical micrographs begs the viewer to look more closely at the image, to inquire as to its origins and how the image was obtained. We all have seen numerous examples (vendor calendars, for example) where optical, electron and scanning probe micrographs are displayed. Generally, these are geared to the seasoned scientist and are available primarily to the scientific community. Why not make such imagery, indeed, why not broadcast such imagery to the general public? Once the attention of the viewer is captured, inquiry-based learning can begin. This is precisely what distinguishes VISUAL from many other image-based programs. Accompanying each image is an explanation, in nontechnical language, of the science underlying the image, the methods used to obtain the result and a potential application of the science. The viewer not only can appreciate the beauty of the image but can also come to appreciate the importance of science in their everyday lives. It also represents a challenge to the researcher to convey the underlying science in terms that anyone can understand. Try it some time and you will appreciate the difficulty of this seemingly simple task.

The essential element of the VISUAL program is that the emphasis is not on the artistry of these images, but the science behind them. Students and post-doctoral fellows performing research for the MRSEC routinely obtain images in their pursuit of answers to important scientific questions. VISUAL began by mounting, matting, and framing these images, along with a title and the researcher's name, to produce a gallery intended to promote an awareness of science to the general public. The program has had many surprising benefits. The interest and pride that young researchers have shown in seeing their own work placed on display has been impressive. This excitement 


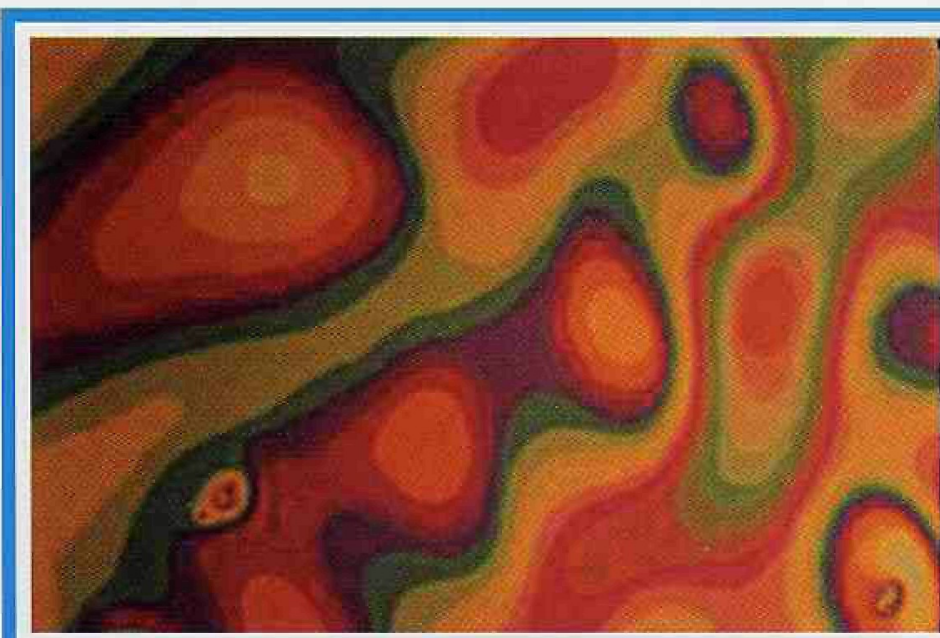

Strag's Leap The color contour map of the mountain range shown in the image was obtain with an optical microscope by reflecting light through a thin film of a block copolymer that formed a layered structure. This layering caused a terracing of the surface where each color corresponds to a specific thickness. From one color to another, the height of the step is about 10 nanometers, about $1,000,000$ times thinner than a sheet of paper. This image was not colorized and the colors seen are exactly what is observed in the microscope. The uniformity of the color in each terrace leads to the remarkable observation that the films thickness is uniform to within a fraction of a nanometer. @University of Massachusetts Amherst, 2003.

has also extended to faculty researchers associated with the program. The imagery that has been produced has resulted in glowing reviews by seasoned scientists and the general public alike.

The UMass MRSEC has several effective and active K-12 educational outreach programs and VISUAL provides a novel complement. Studies have shown that "programs that were able to show a real impact on students and teachers were those that started early..." If the attention and inquisitiveness of the students must be captured initially before the formal education process can begin, then we have no more effective means to accomplish this than a beautiful or mystifying image. While traditional scientific presentations may and do lead to angst for some, VISUAL, through its imagery, has the potential to tunnel through this barrier and influence future career paths.

In addition to placing imagery onto paper or canvas for display, VISUAL is expanding into the area of inkjet printing on textiles in a collaborative effort with the University of Massachusetts Dartmouth. Even here, VISUAL has potentially opened new horizons for outreach to the public and to research areas in textiles.

The University of Massachusetts Dartmouth Textile Science program is researching ways to optimize inkjet print of images onto fabrics. The resolution and quality of the image depends upon the manner in which inks will spread on a surface. The bleeding of colors in garments is a direct consequence of the ability of inks to be held fast to the fibers and the wicking characteristics of the matt of fibers. Like water being pulled into a straw, so too, inks can be drawn between and spread through the fibers in a fabric. The size of the droplets emerging from the printing heads, the density, absorbency, and chemical treatment of the fibers are all issues of current research interests in fiber technology. Thus, educational outreach and fiber research share a common goal and pose a unique opportunity to gain on multiple fronts.

Transferring imagery to textiles opens new approaches to convey the excitement of science to the average person. Imagine if the popularity of such textiles could achieve the same level of success as Jerry Garcia ties, it would significantly elevate the awareness of scientific research in this country in a subtle, yet, lasting way.
We enjoy the honor of being able to perform scientific research and of being continuously stimulated intellectually in the pursuit of science while being supported by the taxes of fellow citizens. We are, therefore, under the obligation that Representative Ehlers emphasized in his congressional report, "to strengthen the relationship between science and the society that supports it," and "to increase efforts in education at all levels." In our opinion, VISUAL is an effective pathway to achieving these goals.

\section{Acknowledgments.}

We thank P. Calvert and Y. Kim for discussions on inkjet printing on textiles, the students and postdoctoral fellows in the MRSEC program, and NSF for support (DMR0213695).

\section{References}

1. Unlocking Our Future: Toward a New National Science Policy, Committee on Science, House. Congress, 1998.

2. Materials in a New Era: Proceedings of the 1999 Solid State Sciences Committee Forum, Solid State Sciences Committee, National Research Council, 1999.

3. G.D. Nelson, 2003, Remarks on the Release of the NAEP 2000 Science Assessment Results, American Association for the Advancement of Science,

4. Pursuing Excellence: Comparisons of International Eighth-Grade Mathematics and Science Achievement from a U.S. Perspective, 1995 and 1999, Office of Educational Research and Improvement, U.S. Department of Education, NCES 2001-028, Decenber 2000.

5. Freundlich, N. From Sputnik to TIMSS: Reforms in Science Education Make Headway Despite Setbacks, The Harvard Education Letter, Vol. 14, October 1998.

* If you would like more information or to become involved in the program, feel free to contact Linda Strzegowski, VISUAL Director, Polymer Science and Engineering Department, University of Massachusetts, Amherst, MA 01003 or by email (mrsec@polysci.umass.edu).

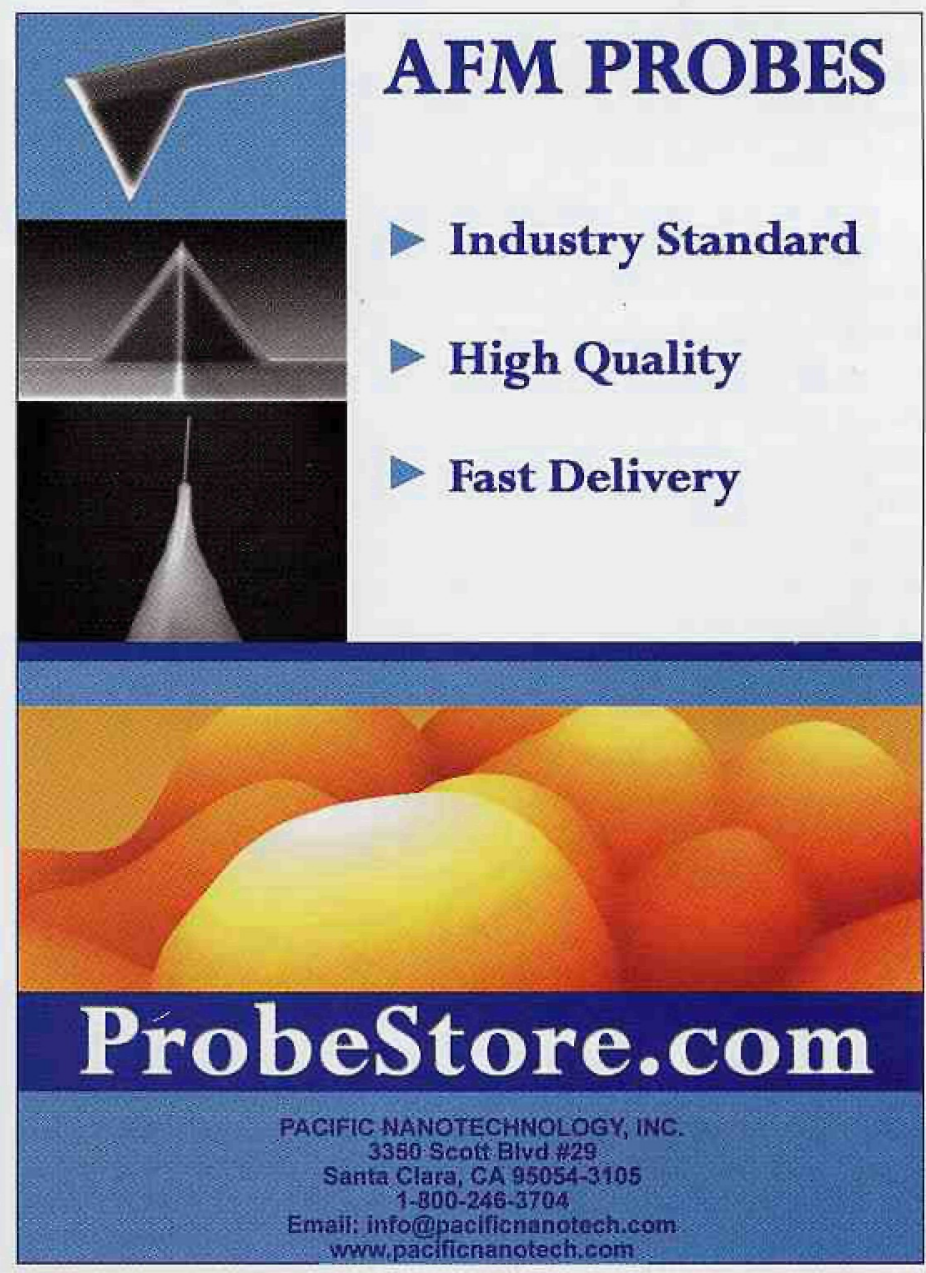

